\title{
Protocolos de rastreamento para o diagnóstico precoce do câncer de pulmão: passado, presente e futuro"
}

\author{
Thamine Lessa E.S. Andrade ${ }^{1}$, Soria Ramos-Barbosa ${ }^{2}$, Jorge L. Pereira-Silva ${ }^{3}$
}

O carcinoma brônquico é, de todos, o de maior letalidade, responsabilizando-se, anualmente, por maior número de óbitos do que aqueles decorrentes do câncer do cólon, mama e próstata juntos. Seguindo seu curso natural, mais de 50\% dos pacientes têm metástases a distância e somente 20 a $25 \%$ são potencialmente ressecáveis no momento do diagnóstico, com perspectiva de sobrevida em cinco anos de apenas 14\%. Os protocolos de rastreamento, baseados em radiografias do tórax e citologia do escarro, realizados há 30 anos com o intuito de estabelecer o diagnóstico precoce, mostraram maior índice de ressecabilidade e melhores taxas de sobrevida, porém sem causar impacto na redução da mortalidade específica. Nos últimos anos, com o advento da tomografia computadorizada helicoidal de baixa dose e de novas técnicas para análise das secreções respiratórias e da mucosa brônquica, com o potencial para identificar casos de câncer de pulmão em fases mais precoces de sua evolução natural, os protocolos de rastreamento voltam a despertar o interesse. Os

autores revisam os protocolos de rastreamento realizados no passado, assim como analisam os estudos prospectivos mais recentes e discutem as perspectivas futuras, destacando suas principais limitações, os problemas metodológicos no seu delineamento e principais vieses que comprometem a interpretação dos resultados. (J Pneumol 2002;28(5):294-301)

\section{Screening for lung cancer: past, present and future}

Lung cancer is the leading cause of death from cancer. More people die each year of bronchial carcinoma than of colon, breast, and prostate cancer combined. More than $50 \%$ of the patients will have distant metastases at diagnosis and only 20-25\% of these will be localized and potentially resectable, with a five-year survival of $14 \%$. Prior chest radiographs and sputum cytology studies lead to clinically meaningful improvements in stage distribution, resectability and survival, but no disease-specific mortality reductions have been demonstrated. More recently, these techniques have evolved to those of screening by low-dose spiral computed tomography and by the use of specific biomarkers for early detection, thus bringing back interest in lung cancer screening. The authors review screening for lung cancer made in the past, analyze more recent prospective studies, and the prospects for the future, and they point to their main limitations, methodological problems in design, major biases, all of which may invalidate the interpretation of results.

* Trabalho realizado no Centro de Enfermidades Respiratórias do Hospital Universitário Professor Edgard Santos, Faculdade de Medicina - Universidade Federal da Bahia, Salvador, BA.

1. Pneumologista. Preceptora voluntária.

2. Médica Residente de Pneumologia.

3. Professor Adjunto Doutor. Supervisor do Programa de Residência Médica em Pneumologia.

Endereço para correspondência - Jorge Pereira, Av. Juracy Magalhães Jr., 2.096, sala 511, Centro Médico Aliança, Rio Vermelho 40295-140 - Salvador, BA. Tel. (71) 350-4653; fax (71) 452-1304; email: cpc-ba@svn.com.br

Recebido para publicação em 4/4/02. Aprovado, após revisão, em 19/6/02.
Descritores - Neoplasias pulmonares. Tabagismo. Carcinoma broncogênico. Protocolos clínicos. Lesão mumular pulmonar.

Key words - Smoking. Lung neoplasms. Bronchogenic carcinoma. Clinical protocols. Coin pulmonary lesion.

\section{INTRODUÇÃO}

O tabagismo é responsável por $90 \%$ dos casos de câncer de pulmão e o risco de mortalidade específica é 22 vezes maior entre os fumantes ${ }^{(1)}$.

No Brasil, em 1995, considerando-se a população de indivíduos com idade maior ou igual a 15 anos, a preva- 
lência de tabagismo em homens foi de 38,2/100.000 e, em mulheres, de 29,3/100.000(2). Segundo os dados do Instituto Nacional do Câncer (INCA) - Ministério da Saú$\mathrm{d} e^{(3)}$, foram registrados $13.579(83,9 / 100.000)$ óbitos por carcinoma brônquico em 1998. Estima-se que o câncer de pulmão tenha atingido 20.835 pessoas (homens: 14.900 e mulheres: 5.935) e causado 15.145 mortes em 2001 (homens: 10.700 e mulheres: 4.445), com taxas brutas de mortalidade de 12,83/100.000 entre homens e 6,86/100.000, entre mulheres ${ }^{(3)}$. No Brasil, no período entre 1979 e 1998, a taxa de mortalidade por câncer de pulmão cresceu 56\% entre os homens e 108\% na população feminina, refletindo o efeito cumulativo provocado pelo aumento no consumo de cigarros pelas mulheres, especialmente a partir dos anos $60^{(3)}$. O câncer de pulmão, entre homens, é o de maior letalidade, em todas as regiões do país, exceto no Nordeste, onde prevalece o câncer da próstata. Em mulheres, o de mama é o de maior letalidade, exceto na região Norte, onde prevalece o câncer do colo do útero(3).

Seguindo seu curso natural, mais que $50 \%$ dos pacientes têm metástases a distância e $20-25 \%$ são potencialmente ressecáveis no momento do diagnóstico, com perspectiva de sobrevida em cinco anos de apenas 14\%(4).

Sua alta incidência e expressivas taxas de morbimortalidade induzem à realização de estratégias que permitam conduzir ao diagnóstico em fases menos avançadas de sua evolução natural, com maior potencial de ressecabilidade e, presumivelmente, maior probabilidade de cura. Os estudos de rastreamento (screening) realizados há 30 anos $^{(5-8)}$, com base em radiografias do tórax e citologia do escarro, demonstraram maior precocidade diagnóstica, maior potencial de ressecabilidade e maiores taxas de sobrevida ${ }^{(9-12)}$. Entretanto, mediante uma análise metodológica mais aprofundada, surpreendentemente, não houve redução na mortalidade ${ }^{(13)}$. Nos últimos anos, com o advento da tomografia helicoidal e de técnicas avançadas para exames do escarro e da mucosa brônquica, o interesse pela implementação de novos protocolos de rastreamento, visando a identificação precoce do carcinoma brônquico, com o potencial para reduzir a mortalidade, tem sido revitalizado ${ }^{(14-20)}$.

\section{Critérios que fornecem o suporte para o ras- treamento de determinada neoplasia ${ }^{(19)}$ :}

1) Deve constituir-se em um importante problema de saúde pública, considerando-se diversos parâmetros, tais como morbidade, mortalidade e outras medidas de agravos à saúde da população;

2) Deve haver uma fase pré-clínica, em que possa ser detectado antes do aparecimento de sintomas. O período de latência deve ser suficientemente longo, para que permita a identificação de maior número de tumores ocul-
Siglas e abreviaturas utilizadas neste trabalho

INCA - Instituto Nacional do Câncer

TMGS - Tokyo Metropolitan Government Study

PPNRP - Philadelphia Pulmonary Neoplasm Research Project

VAT - Veterans Administration Trial

SLLCS - South London Lung Cancer Study

NLLCS - North London Lung Cancer Study

ECGS - Erfurt County Germany Study

$\mathrm{NCI}$ - National Cancer Institute

EUA - Estados Unidos da América

CELCDP - Cooperative Early Lung Cancer Detection Program

MLP - Mayo Lung Project

MSKLP - Memorial Sloan-Kettering Lung Project

JHLP - Johns Hopkins Lung Project

CSLCS - Czech Study on Lung Cancer Screening

$\mathrm{NCCH}$ - National Cancer Center Hospital

SU - Shinshu University

$\mathrm{Rx}$ - Radiografia do tórax

TCbd - Tomografia Computadorizada de baixa dose

ELCAP - Early Lung Cancer Action Project

tos. Caso contrário, a necessidade de realização de exames a intervalos muito curtos tornaria o programa de rastreamento impraticável;

3) O tratamento de lesões diagnosticadas na fase préclínica deve ser vantajoso, em relação à terapêutica dirigida a pacientes sintomáticos e presumivelmente com doença mais avançada;

4) Os testes usados no rastreamento devem ter níveis aceitáveis de custo e eficácia diagnóstica;

5) Esses testes, e a investigação subseqüente de casos identificados, devem ter bom nível de aceitação por parte dos indivíduos e dos planos de saúde.

\section{PERSPECTIVA HISTÓRICA}

Dez estudos prospectivos, utilizando controles radiológicos periódicos, com ou sem exame citopatológico do escarro, foram conduzidos entre 1951 e 1975(21-29). Exceto no Tokyo Metropolitan Government Study (TMGS)(25), foram incluídos apenas indivíduos do sexo masculino.

\section{Estudos não-randomizados}

Quatro estudos não-randomizados e não-controlados foram realizados. O Philadelphia Pulmonary Neoplasm Research Project (PPNRP)(21-23) e o Veterans Administration Trial (VAT)(24) não conseguiram demonstrar qualquer benefício do rastreamento, enquanto o TMGS (Tokyo Metropolitan Government Study)(25) e o SLLCS (South London Lung Cancer Study)(26) sugeriram alguma melhora na taxa de sobrevida, mas a mortalidade específica não foi adequadamente avaliada. Os resultados desanimadores no PPNRP (21-23) e no VAT ${ }^{(24)}$ devem-se, pelo menos em parte, à idade avançada da população estudada, ao grande número de co-morbidades, menor potencial 
de ressecabilidade e maior mortalidade perioperatória ${ }^{(30)}$ (Tabela 1).

O TMGS(25) e o $\operatorname{SLLCS}^{(26)}$ são grandes estudos não-controlados, cujo acompanhamento foi feito mediante controles radiológicos periódicos. Ambos demonstraram alto percentual de ressecabilidade e animadoras taxas de sobrevida, sobretudo quando comparadas, retrospectivamente, com controles contemporâneos de outros grupos de pacientes (Tabela 1).

Dois outros estudos não-randomizados, controlados, também foram realizados nesse período. O North London Lung Cancer Study (NLLCS) ${ }^{(27,28)}$, em 1959, comparou um grupo de estudo, para o qual foram oferecidos controles radiológicos semestrais por três anos, com um grupo-controle, submetido a exames radiológicos apenas no início e no fim do período do estudo. O Erfurt County Germany Study (ECGS)(29), em 1972, comparou um grupo submetido a controles radiológicos semestrais, com um grupo-controle em que a periodicidade ocorreu a cada um ou dois anos. Em ambos, ficou demonstrada a maior precocidade do diagnóstico, maior índice de ressecabilidade e sobrevida mais longa, nos grupos submetidos ao rastreamento. Entretanto, em nenhum deles se conseguiu demonstrar redução significativa da mortalidade, embora, em ambos, a incidência de câncer de pulmão tenha sido maior nos grupos em estudo.

\section{Estudos randomizados}

O maior impacto ocorreu nos anos 70, quando o National Cancer Institute (NCI) ${ }^{(9)}$ patrocinou três estudos prospectivos, randomizados, controlados, nos Estados Unidos da América (EUA), denominado Cooperative Early Lung Cancer Detection Program (Tabela 2). No Mayo Lung Project (MLP) (1971-1983)(7), foram randomizados 9.211 indivíduos fumantes do sexo masculino. O grupo em estudo $(n=4.618)$ foi convidado a submeter-se a radiografias do tórax e exames citopatológicos do escarro a cada quatro meses, enquanto o grupo-controle $(n=4.593)$

TABELA 1

Estudos de rastreamento não-randomizados e não-controlados

\begin{tabular}{lcccccc}
\hline Estudo & $\begin{array}{c}\text { Ano de } \\
\text { início }\end{array}$ & $\begin{array}{c}\text { Idade } \\
(\mathbf{a n o s})\end{array}$ & $\begin{array}{c}\text { Participantes } \\
\mathbf{( n )}\end{array}$ & $\begin{array}{c}\text { Câncer } \\
(\mathbf{n})\end{array}$ & $\begin{array}{c}\text { Ressecabilidade } \\
(\mathbf{\%})\end{array}$ & $\begin{array}{c}\text { Sobrevida } \\
(\mathbf{\%})\end{array}$ \\
\hline PPNRP $^{(21-23)}$ & 1951 & $>45$ & 6.136 & 121 & 35 & $8(5$ anos $)$ \\
VAT $^{(24)}$ & 1958 & 62,8 (média) & 14.607 & 73 & 36 & $17(32$ meses $)$ \\
TMGS $^{(25)}$ & 1953 & Todas & 1.871 .374 & 193 & 56 & $44(5$ anos $)$ \\
SLLCS $^{(26)}$ & 1955 & $>45$ & 67.400 & 234 & 56 & $18(4$ anos $)$ \\
\hline
\end{tabular}

TABELA 2

Estudos de rastreamento prospectivos, randomizados e controlados

\begin{tabular}{|c|c|c|c|c|c|c|c|c|c|}
\hline Estudo & $\begin{array}{l}\text { Ano de } \\
\text { início }\end{array}$ & $\begin{array}{l}\text { Idade } \\
\text { (anos) }\end{array}$ & $\begin{array}{l}\text { Part. } \\
\text { (n) }\end{array}$ & $\begin{array}{c}\text { Câncer } \\
\text { (n) }\end{array}$ & $\begin{array}{l}\text { Preval. } \\
\text { (n) }\end{array}$ & $\begin{array}{c}\text { Incid. } \\
\text { (n) }\end{array}$ & $\begin{array}{c}\text { Ressec. } \\
(\%)\end{array}$ & $\begin{array}{c}\text { Sobrevida } \\
(\%)\end{array}$ & $\begin{array}{c}\text { Mort. } \\
(1.000 / \text { ano })\end{array}$ \\
\hline MSKLP(6) & 1974 & $>45$ & $\begin{array}{l}5.072 \text { (exp) } \\
4.968 \text { (cont) }\end{array}$ & $\begin{array}{l}144 \\
144\end{array}$ & $\begin{array}{l}30 \\
23\end{array}$ & $\begin{array}{l}114 \\
121\end{array}$ & $\begin{array}{l}53 \\
51\end{array}$ & $\begin{array}{l}35 \text { (5 anos) } \\
35 \text { (5 anos) }\end{array}$ & $\begin{array}{l}2,7 \\
2,7\end{array}$ \\
\hline$J H L P^{(5)}$ & 1973 & $\geq 45$ & $\begin{array}{l}5.226 \text { (exp) } \\
5.161 \text { (cont) }\end{array}$ & $\begin{array}{l}194 \\
202\end{array}$ & $\begin{array}{l}39 \\
40\end{array}$ & $\begin{array}{l}155 \\
162\end{array}$ & $\begin{array}{l}47 \\
44\end{array}$ & $\begin{array}{l}20 \text { (8 anos) } \\
20 \text { (8 anos) }\end{array}$ & $\begin{array}{l}3,4 \\
3,8\end{array}$ \\
\hline$M L P^{(7)}$ & 1971 & $>45$ & $\begin{array}{c}10.933 \text { (prev) } \\
4.618 \text { (exp) } \\
4.593 \text { (cont) }\end{array}$ & $\begin{array}{l}- \\
- \\
-\end{array}$ & $\begin{array}{l}91 \\
- \\
-\end{array}$ & $\begin{array}{l}- \\
206 \\
160\end{array}$ & $\begin{array}{l}54 \\
46 \\
32\end{array}$ & $\begin{array}{l}40 \text { (5 anos) } \\
33 \text { (5 anos) } \\
15 \text { (5 anos) }\end{array}$ & $\begin{array}{c}- \\
3,2 \\
3,0\end{array}$ \\
\hline Czech $^{(8)}$ & 1976 & $40-64$ & $\begin{array}{c}6.364 \text { (prev) } \\
3.172 \text { (exp- } \\
\text { 3a) } \\
3.174 \text { (cont- } \\
3 \mathrm{a} \text { ) } \\
3.172 \text { (exp- } \\
6 \mathrm{a} \text { ) } \\
3.174 \text { (cont- } \\
6 \mathrm{a} \text { ) }\end{array}$ & $\begin{array}{l}- \\
- \\
- \\
- \\
-\end{array}$ & 19 & $\begin{array}{r}- \\
36 \\
19 \\
108 \\
82\end{array}$ & $\begin{array}{l}- \\
25 \\
16 \\
- \\
-\end{array}$ & $\begin{array}{c}28 \text { (5 anos) } \\
24 \text { (5 anos) } \\
0 \text { (5 anos) } \\
- \\
-\end{array}$ & $\begin{array}{l}- \\
1,7 \\
1,5 \\
3,6 \\
2,6\end{array}$ \\
\hline
\end{tabular}


foi aconselhado, porém sem qualquer esforço para aumentar a taxa de adesão, a realizar radiografias do tórax e citologia do escarro anualmente. Como ponto de partida, antes da randomização, um estudo de prevalência identificou 91 casos de câncer $(8,3 / 1.000)$, dos quais a metade foi considerada passível de ressecção. A taxa de sobrevida em cinco anos, na amostra de prevalência, foi de 40\%. No transcorrer do estudo, 206 casos de carcinoma brônquico foram diagnosticados no grupo estudado $e$ 160 no grupo-controle (incidência). A citologia do escarro identificou apenas 18 casos. A adesão do grupo em estudo foi de 75\%, embora tenha havido uma "contaminação" significativa no grupo-controle, quando 53\% dos indivíduos mostraram-se aderentes aos exames radiológicos no último ano do estudo. Embora a sobrevida em cinco anos no grupo em estudo tenha sido maior (33\% x $15 \%)$, não houve diferença estatisticamente significante na mortalidade por câncer de pulmão $(3,2 \times 3,0)$, expressa pelo número de mortes por 1.000 indivíduos estudados/ano ${ }^{(7)}$.

Os outros dois estudos prospectivos, do NCI, avaliaram a vantagem adicional de incorporar a citologia do escarro aos exames radiográficos. No Memorial Sloan-Kettering Lung Project (MSKLP) (1974-1982)(6), foram randomizados 10.040 homens, com idade igual ou maior que 45 anos, fumantes de 20 cigarros por dia, em média. O grupo em estudo $(n=5.072)$ foi submetido a radiografias do tórax anuais e citologia do escarro a cada quatro meses, enquanto o grupo-controle $(\mathrm{n}=4.968)$ foi submetido apenas ao controle radiológico anual. Os autores conclúram que a radiografia do tórax foi superior à citologia do escarro na detecção precoce do câncer de pulmão(6). Quarenta por cento dos casos foram classificados no estádio I, e 2/3 deles não morreram por câncer de pulmão. A sobrevida em cinco anos do grupo em estudo foi maior $(35 \% \times 13 \%)^{(6)}$. Entretanto, considerando-se o objetivo primário do estudo, os autores concluíram que a incorporação da citologia do escarro não produziu impacto na redução da mortalidade por câncer de pulmão.

O Johns Hopkins Lung Project (JHLP) (1973-1982)(5) mostra um delineamento semelhante ao do MSKLP(6):
10.387 indivíduos fumantes, com idade igual ou maior que 45 anos, foram randomizados e alocados em um grupo de estudo $(\mathrm{n}=5.226)$, submetido a controles radiológicos anuais e citologia do escarro a cada quatro meses, enquanto o grupo-controle $(\mathrm{n}=5.161)$ se submeteu apenas ao acompanhamento radiológico anual. Foram identificados 194 casos de câncer de pulmão no grupo estudado e 202 no grupo-controle. Metade deles foi considerada interval cancers (cânceres que surgem e se manifestam por sintomas no intervalo entre os testes de rastreamento), não-identificados no rastreamento. Dentre esses, apenas $17 \%$ foram classificados no estádio I, quando comparados com os $57 \%$ identificados no rastreamento(5). Nenhuma diferença foi observada nos diversos parâmetros avaliados em ambos os grupos, chegando-se à conclusão de que a inclusão da citologia do escarro não produziu impacto na redução da mortalidade por câncer de pulmão $(3,4 \times 3,8)^{(5)}$.

Além desses estudos realizados nos EUA, na Tchecoslováquia, o Czech Study on Lung Cancer Screening (CSLCS) $(1976-1983)^{(8)}$ randomizou 6.364 indivíduos fumantes, do sexo masculino, com idade compreendida entre 40 e 64 anos. O grupo em estudo foi convidado a submeter-se a controles radiológicos semestrais, enquanto o grupo-controle foi submetido ao exame radiográfico ao final de três anos. A partir daí, todos os indivíduos, independentemente do grupo de origem, foram submetidos a controles radiológicos anuais, nos três anos subseqüentes, ao longo dos seis anos do estudo( ${ }^{(8)}$. O tempo de sobrevida foi maior no grupo em estudo, para os casos diagnosticados nos primeiros três anos de acompanhamento, embora não tenha diferido na taxa de mortalidade $(1,7 \times 1,5)^{(8)}$.

\section{Perspectivas ATUAIS E FUtURAS}

Os estudos realizados no passado, com base em controles radiológicos do tórax e na citologia do escarro(5-8), demonstraram resultados surpreendentemente desanimadores ${ }^{(13)}$. Trinta anos mais tarde, desde a realização dos estudos randomizados e controlados, com o advento de novos recursos de diagnóstico por imagem e de técnicas

TABELA 3

Estudos de rastreamento utilizando TC-helicoidal

\begin{tabular}{lccccccr}
\hline Estudo & $\begin{array}{l}\text { Idade } \\
\text { (anos) }\end{array}$ & $\begin{array}{c}\text { RX } \\
\text { (freqüência) }\end{array}$ & $\begin{array}{c}\text { TC } \\
\text { (freqüência) }\end{array}$ & $\begin{array}{c}\text { Participantes } \\
(\mathbf{n})\end{array}$ & $\begin{array}{c}\text { Nódulos } \\
\mathbf{n}(\%)\end{array}$ & $\begin{array}{c}\text { Câncer } \\
\mathbf{n}(\%)\end{array}$ & $\begin{array}{c}\text { Estádio I } \\
\mathbf{n}(\%)\end{array}$ \\
\hline $\mathrm{NCCH}^{(32)}$ & $>50$ & Semestral & Semestral & 1.369 & - & $15(0,43)$ & $14(93)$ \\
$\mathrm{SU}^{(33)}$ & $>40$ & Anual & Anual & 5.483 & - & $19(0,35)$ & $16(84)$ \\
$\mathrm{ELCAP}^{(34)}$ & $>60$ & Anual & Anual & 1.000 & $233(23)$ & $27(2,7)$ & $23(85)$ \\
$\mathrm{MC}^{(15)}$ & $\geq 50$ & $\mathrm{NR}$ & Anual & 1.520 & $782(51)$ & $15(1)$ & $9(60)$ \\
Münster $^{(15)}$ & $\geq 40$ & - & Anual & 919 & - & $13(1,4)$ & $8(62)$ \\
\hline
\end{tabular}


avançadas para análise do escarro e da mucosa brônqui$\mathrm{ca}$, ressurge o interesse por métodos de rastreamento para o diagnóstico precoce do carcinoma brônquico ${ }^{(31)}$.

Os dados de prevalência de três estudos foram recentemente publicados (Tabela 3). Dois deles, não-randomizados, realizados no Japão, no National Cancer Center Hospital $(\mathrm{NCCH})^{(32)}$ e na Shinshu University (SU) ${ }^{(33)}$, quando foram utilizadas radiografias do tórax $(\mathrm{Rx})$, tomografia computadorizada de baixa dose (TCbd) e exames de escarro resultantes de três dias de coleta. No $\mathrm{NCCH}^{(32)}$ ( $\mathrm{n}=$ 1.369), as TCbd (grupo em estudo) e as radiografias do tórax (grupo-controle) tiveram periodicidade semestral, identificando-se 15 casos de câncer (11 deles com radiografias consideradas normais), 14 dos quais (93\%) no estádio I(32). Nesse estudo, a sobrevida em cinco anos foi de $78 \%$. Enquanto no $\mathrm{SU}^{(33)}(\mathrm{n}=5483)$ os controles foram realizados anualmente, identificando-se 19 casos, 16 (84\%) no estádio I.

Um terceiro estudo, também não-randomizado, o Early Lung Cancer Action Project (ELCAP) ${ }^{(34)}$, arrolou 1.000 indivíduos fumantes de alto risco, com mais de 60 anos de idade, submetidos a Rx e TCbd do tórax com periodicidade anual. Com a TCbd, foram identificados 233 (23\%) participantes com nódulos não-calcificados, 27 (2,7\%) dos quais de caráter maligno ${ }^{(34)}$. Vinte e seis deles foram ressecados e 23 (85\%) classificados no estádio I. Em 15 dos 27 casos de câncer de pulmão, o diâmetro dos nódulos foi $\leq 10 \mathrm{~mm}^{(34)}$. Em contrapartida, a radiografia do tórax identificou 68 nódulos, sete deles de natureza maligna, apenas quatro no estádio I(34).

Outro estudo, realizado na Universidade de Münster, na Alemanha ${ }^{(15)}$, com 919 participantes, permitiu a identificação de 13 casos de câncer de pulmão (prevalência de $1,4 \%)$, oito deles (62\%) no estádio I (Tabela 3).

Em face da necessidade de realização de novos estudos prospectivos, randomizados, controlados, o American College of Radiology Imaging Network ${ }^{(35)}$, sob o patrocínio do National Cancer Institute, delineou um estudo que compreende o acompanhamento de 7.000 indivíduos de alto risco, com o qual se projeta uma taxa de sobrevida em cinco anos de $80 \%$, mediante a realização anual de TCbd (grupo em estudo) ou Rx do tórax (grupocontrole). Ademais, o NCI considera a possibilidade de conduzir um estudo envolvendo 88.000 participantes, presumivelmente capaz de demonstrar redução de 20\% na mortalidade $e^{(15)}$.

Os estudos atuais confirmam que a TC helicoidal com baixa dose de radiação (TCbd) é mais sensível do que o Rx do tórax na detecção precoce de nódulos pulmonares $e$ que uma parcela destes é maligna ${ }^{(17,18)}$. Ao compararmos os resultados dos estudos com TC com os que usaram Rx do tórax e citologia do escarro, a TC identifica maior número de casos de carcinoma brônquico (27/1.000 vs.
9,1 a 7,6/1.000) com maior probabilidade de diagnóstico precoce ${ }^{(15)}$. Alguns outros estudos estão em andamento, embora apenas os resultados preliminares de prevalência sejam conhecidos. Em 1999, iniciou-se um estudo não-randomizado, na Mayo Clinic( ${ }^{(15)}$, que envolveu 1.520 indivíduos fumantes ou ex-tabagistas. No início, todos foram submetidos a TCbd e a exame citopatológico do escarro, devendo-se repetir os exames anualmente por três anos consecutivos. Os dados preliminares revelaram 15 casos de câncer de pulmão, $60 \%$ deles considerados em estádio precoce. Infelizmente, em $51 \%$ de toda a amostra, foram identificados nódulos pulmonares de natureza indeterminada, exigindo acompanhamento tomográfico a cada três meses ${ }^{(18)}$.

Além dos métodos de diagnóstico por imagem, outros recursos envolvendo amostras do escarro, do lavado broncoalveolar e de biópsias da mucosa brônquica (inclusive obtidas por broncoscopia fluorescente), vêm sendo aplicados, embora nenhum deles tenha sido testado, em larga escala, como método isolado de rastreamento(36-45). Técnicas de imunohistoquímica de biópsias da mucosa brônquica, a pesquisa de mutações genéticas (p53 e Kras), a determinação da atividade da telomerase e de anormalidades na metilação do DNA têm sido testadas, porém com sensibilidade e especificidade baixas, sobretudo nos nódulos periféricos ${ }^{(42-45)}$. Além disso, o caráter invasivo da investigação constitui-se em importante empecilho para que seja incorporada em programas de saúde pública.

\section{Problemas METOdológicos E PRINCIPAIS LIMITAÇÕES DOS PROTOCOLOS DE RASTREAMENTO}

Os protocolos de rastreamento para o diagnóstico precoce do carcinoma brônquico devem obedecer a critérios rígidos de delineamento e de análise dos resultados, procurando evitar os vieses de interpretação.

Lead-time $\boldsymbol{e}^{(15,19)}$ : Designa o intervalo de tempo entre o momento em que o nódulo seria passível de detecção pelo rastreamento e o momento em que se manifestaria por sinais e/ou sintomas. A partir da entrada no estudo, considerando-se que o diagnóstico é mais precoce no grupo estudado (identificado por testes sistemáticos, realizados a intervalos predefinidos) do que no grupo-controle (alertado pelo quadro clínico), há aparente melhora da sobrevida (tempo decorrido entre o momento do diagnóstico e a ocorrência do óbito), embora a mortalidade não difira entre os grupos (lead-time bias).

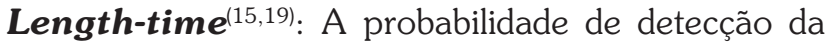
doença está relacionada à taxa de crescimento da neoplasia. Os tumores de crescimento rápido e de comportamento mais agressivo necessitariam de controles sistemáticos mais freqüentes, considerando-se o intervalo entre a possível detecção e a ocorrência de sintomas. Portanto, 
se os controles periódicos não forem realizados a intervalos muito curtos, pacientes com tumores mais agressivos tenderiam a apresentar-se com sintomas. Em contrapartida, os tumores de crescimento mais lento demonstram um período potencial de rastreamento mais longo, podendo ser identificados ainda na ausência de sintomas. Como resultado, maior proporção de tumores indolentes é identificada no grupo em estudo, causando a impressão de aparente melhora no tempo de sobrevida (length-time bias).

Overdiagnosis bias $^{(15,19)}$ : Corresponde à forma extrema de length-time bias. A detecção de maior número de tumores muito indolentes no grupo estudado que, provavelmente, não iriam expressar-se clinicamente, produz um aumento aparente no número de casos de câncer de pulmão e na sobrevida. Esta hipótese tem sido muito contestada(46): o câncer de pulmão, por ser o mais letal $e$ responsável por maior número de óbitos do que tem sido atribuído ao câncer de mama, próstata e colorretal, juntos, dificilmente teria um comportamento benigno ou um curso excessivamente indolente. Embora em estudos de autópsia não-selecionados fique demonstrada a presença inusitada de câncer de próstata em até 30\% dos indivíduos com mais de 50 anos de idade ${ }^{(47)}$, há poucas evidências de que o mesmo ocorra no pulmão. Em um estudo com 3.286 autópsias $^{(48-50)}$, houve o achado inesperado de câncer de pulmão em apenas 26 casos. Ademais, em sua maioria, não havia evidências de que os tumores estivessem em fase latente ou que fossem clinicamente irrelevantes (58\% tinham metástases regionais ou a distância) e, presumivelmente, iriam morrer pela própria neoplasia ${ }^{(48-50)}$.

Patient self-selection ${ }^{(15,19)}$ : Pacientes que aderem a programas de rastreamento são, presumivelmente, mais conscienciosos, estão mais atentos à presença de novos sintomas, costumam cuidar melhor de sua saúde e são aparentemente mais saudáveis. Essas características criam um viés de auto-seleção nos estudos não-randomizados.

Stage shift ${ }^{(15,19)}$ : embora no grupo submetido ao rastreamento sistemático, sobretudo com TCbd, seja detectado maior número de casos no estádio I, não se observa uma verdadeira mudança no estadiamento. Para tanto, seria necessário haver não somente maior número de casos diagnosticados em etapa precoce de sua evolução natural, como também, simultaneamente, que se produzisse uma diminuição do número de casos diagnosticados em fase avançada, com o potencial para determinar redução significativa da taxa de mortalidade.

Radiografias do tórax vs. tomografia computadorizada helicoidal ${ }^{(51-56)}$ : Os estudos realizados no passado demonstraram as limitações da radiografia do tórax na identificação precoce de nódulos pulmonares. No Mayo Lung Project, em uma análise retrospectiva, 90\% dos carcinomas periféricos e 75\% dos peri-hilares, já estavam presentes em radiografias anteriores ${ }^{(51)}$. Quekel et al. ${ }^{(52)}$ demonstraram que 19\% dos casos de carcinomas periféricos escaparam à identificação radiológica (diâmetro médio de $16 \mathrm{~mm})$. Sone et al. ${ }^{(53)}$ compararam a acurácia da radiografia do tórax com a tomografia computadorizada helicoidal, em 44 casos de câncer de pulmão identificados no exame tomográfico. As radiografias foram omissas em 77\% (34 de 44) dos casos, sobretudo em nódulos com o diâmetro $\leq 20 \mathrm{~mm}^{(53)}$. Kaneko et al. ${ }^{(54)}$, mediante radiografias do tórax e TCbd, detectaram 31 casos de carcinoma brônquico periférico em 9.993 pacientes (0,3\%). Dos 31 casos, 24 (77\%) foram identificados pela TCbd e não foram vistos às radiografias do tórax. Em 1999, Henschke et al.(56), em uma análise de prevalência, em que foram estudados 1.000 indivíduos, a TC revelou 27 casos de carcinoma brônquico, dos quais apenas sete podiam ser vistos nas radiografias. Vinte e dois dos 27 casos de câncer eram estádio IA. Em 15 dos 27, o diâmetro dos nódulos era menor ou igual a $10 \mathrm{~mm}$.

Indubitavelmente, a TC helicoidal é capaz de identificar nódulos pulmonares com diâmetro $\leq 15 \mathrm{~mm}$, usualmente inacessíveis ao exame radiográfico convencional. Cerca de $80 \%$ dos casos de carcinoma brônquico identificados com essas dimensões são estádio IA, enquanto, na prática diária, apenas 15-20\% dos casos são assim classifica$\operatorname{dos}^{(14)}$

A maior limitação da TC deve-se à identificação de grande número de nódulos indeterminados (maior sensibilidade em detrimento de menor especificidade)(15).

Pequenos nódulos vs. diagnóstico precoce ${ }^{(57-60)}$ : Segundo o sistema de estadiamento atualmente empregado ${ }^{(61)}$, a sobrevida em cinco anos de pacientes com estádio patológico T1N0M0 (nódulo isolado) é de 67\%, contra os $57 \%$ daqueles classificados em T2NOM0 (massa isolada) ${ }^{(14,61)}$. Entretanto, ainda não se conseguiu demonstrar que os pequenos nódulos $(<30 \mathrm{~mm})$ detectados pela TCbd, e fora do limite de resolução das radiografias de tórax, tenham menor capacidade de produzir metástases e que se associem a melhor prognóstico ${ }^{(57)}$. Nódulos identificados com o diâmetro menor ou igual a $20 \mathrm{~mm}$ não estão necessariamente em uma etapa precoce de seu desenvolvimento, uma vez que em $20 \%$ dos casos são classificados como $\mathrm{N}^{(16)}$. Diminutos nódulos, com 10 a $20 \mathrm{~mm}$ de diâmetro, são capazes de disseminar-se, mesmo em uma etapa precoce da angiogênese $e^{(15)}$. Não há dados que sustentem que um nódulo pulmonar maligno com $5 \mathrm{~mm}$ de diâmetro (cerca de $10^{8}$ células) tenha melhor prognóstico do que outro com $10 \mathrm{~mm}$ ( $10^{9}$ células) ou, até mesmo, do que outro com $30 \mathrm{~mm}\left(2,7 \times 10^{10}\right.$ células)(15). Independentemente do tamanho, todos esses nódulos encontram-se em fase avançada de sua história natural, uma vez que, no momento do óbito, o carcino- 
ma brônquico habitualmente compreende cerca de $10^{12}$ células ${ }^{(15)}$. Em um estudo recente, envolvendo 510 pacientes com câncer de pulmão (T1NOM0), não se conseguiu demonstrar uma correlação significativa entre as pequenas dimensões do nódulo no momento do diagnóstico e a sobrevida. O desfecho de indivíduos com massas com $30 \mathrm{~mm}$ de diâmetro foi igual ao daqueles com nódulos de $10 \mathrm{~mm}^{(57)}$.

Ademais, alguns estudos demonstram que $60 \%$ dos indivíduos classificados clínica e radiologicamente no estádio I sucumbem ao câncer de pulmão dentro de cinco anos, a despeito do tratamento apropriado ${ }^{(61)}$. Isso sugere que, em grande número de casos, haja doença oculta disseminada no momento de sua apresentação.

O real significado clínico da identificação de pequenos nódulos no rastreamento não é conhecido e seu impacto na redução da taxa de mortalidade aguarda novas investigações.

Nódulos indeterminados: A TC helicoidal, sem contraste e com baixa intensidade de radiação, inserida nos protocolos de rastreamento do câncer de pulmão, demonstra maior sensibilidade do que as radiografias de tórax, na detecção precoce de nódulos pulmonares, embora com menor especificidade. Em uma análise de prevalência, na qual 1.000 indivíduos fumantes, com mais de 60 anos, foram submetidos ao protocolo ELCAP(56), a TCbd identificou nódulos não-calcificados em 233 pacientes (23\%). Apenas 27 deles (12\%) eram malignos. A identificação de nódulos de etiologia indeterminada gera maior custo $e$ apreensão, por não haver uma conduta padronizada na condução desses casos.

Análise de custo-efetividade ${ }^{(19)}$ : Resulta do quociente entre o gasto envolvido no processo de rastreamento $e$ os eventuais benefícios obtidos em modelos predefinidos. Compara os custos (valor monetário) necessários para alcançar um determinado desfecho de saúde. Embora diversos parâmetros possam ser analisados, isoladamente ou em conjunto, tais como taxa de sobrevida, morbidade e qualidade de vida, os protocolos visam estabelecer o diagnóstico precoce para que, mediante uma intervenção (habitualmente, a ressecção da lesão), seja possível reduzir a mortalidade. Considerando-se que, até então, os estudos não conseguiram demonstrar a redução na taxa de mortalidade, não é possível comprovar a custo-efetividade dos protocolos de rastreamento do câncer de pulmão.

\section{CONCLUSÃO}

Com base no conhecimento científico atual, protocolos de rastreamento para o diagnóstico precoce do carcinoma brônquico não devem ser incorporados aos programas de saúde pública. Entretanto, a realização de novos estudos, que utilizem os recursos tecnológicos atualmen- te disponíveis, com o potencial para solucionar os problemas metodológicos existentes, é altamente recomendável. O controle do tabagismo, ainda que o impacto possa manifestar-se apenas a longo prazo (para os ex-fumantes, mesmo com 20 a 30 anos de abstinência, o risco permanece maior do que para os que nunca fumaram)(57), é a medida mais efetiva na redução da incidência e da mortalidade por câncer de pulmão.

\section{REFERÊNCIAS}

1. Centers for Disease Control and Prevention. Smoking-attributable mortality and years of potential life lost - United States, 1990. Morb Mortal Wkly Rep 1993;42:645-8.

2. World Health Organization. Tobacco Control Country Profiles. Smoking Prevalence. Region of the Americas. Brazil, 1995, p. 150-1 [on line]. Available from: http://tobacco.who.int/repository/tld103/ Brazil.pdf

3. INCA-Instituto Nacional de Câncer - Ministério da Saúde - [on line]. Available from: www.inca.org.br/inca/releases/press_estimativas2001. html.

4. Greenlee RT, Murray T, Bolden S, Wingo PA. Cancer statistics, 2000. CA Cancer J Clin 2000;50:7-33.

5. Frost JK, Ball Jr WC, Levin ML, Tockman MS, Baker RR, Carter D, et al. Early lung cancer detection: results of the initial (prevalence) radiologic and cytologic screening in the Johns Hopkins Study. Am Rev Respir Dis 1984;130:549-54.

6. Flehinger BJ, Melamed MR, Zaman MB, Heelan RT, Perchick WB, Martini N. Early lung cancer detection: results of the initial (prevalence) radiologic and cytologic screening in Memorial Sloan-Kettering study. Am Rev Respir Dis 1984;130:555-60.

7. Fontana RS, Sanderson DR, Taylor WF, Woolner LB, Miller WE, Muhm $\mathrm{JR}$, et al. Early lung cancer detection: results of the initial (prevalence) radiologic and cytologic screening in the Mayo Clinic study. Am Rev Respir Dis 1984;130:561-5.

8. Kubik A, Polak J. Lung cancer detection: results of a randomized prospective study in Czechoslovakia. Cancer 1986;57:2427-37.

9. Berlin NI, Buncher CR, Fontana RS, Frost JK, Melamed MR. The National Cancer Institute Cooperative Early Lung Cancer Detection Program: results of the initial screen (prevalence): early lung cancer detection: introduction. Am Rev Respir Dis 1984;130:545-9.

10. Fontana RS, Sanderson DR, Woolner LB, Miller WE, Bernatz PE, Payne WS, et al. The Mayo Lung Project for early detection and localization of bronchogenic carcinoma: a status report. Chest 1975;67:511-22.

11. Tockman MS. Survival and mortality from lung cancer in a screened population: the Johns Hopkins study. Chest 1986;89(Suppl):324S$5 \mathrm{~S}$.

12. Fontana RS, Sanderson DR, Woolner LB, Taylor WF, Miller WE, Muhm JR, et al. Screening for lung cancer: a critique of the Mayo Lung Project. Cancer 1991;67(Suppl):1155-64.

13. Flehinger BJ, Melamed MR. Current status of screening for lung cancer. Chest Surg Clin N Am 1994;4:1-15.

14. Aberle DR, Gamsu G, Henschke CI, Naidich DP, Swensen SJ. A consensus statement of the Society of Thoracic Radiology: screening for lung cancer with helical computed tomography. J Thorac Imaging 2001; 16:65-8.

15. Patz Jr EF, Goodman PC, Bepler G. Screening for lung cancer. N Engl J Med 2000;343:1627-33.

16. Deslauriers J. Should screening for lung cancer be revisited? J Thorac Cardiovasc Surg 2001;121:1031-2. 
17. Jett JR. Spiral computed tomography screening for lung cancer is ready for prime time. Am J Respir Crit Care Med 2001;163:812-5.

18. Patz Jr EF, Goodman PC. Low-dose spiral computed tomography screening for lung cancer: not ready for prime time. Am J Respir Crit Care Med 2001;163:812-5.

19. Eyre HJ, Smith RA, Mettlin CJ. Cancer screening and early detection. In: Bast Jr RC, Kufe DW, Pollock RE, Weichselbaum RR, Holland JF, Frei E. Cancer medicine. $5^{\text {th }}$ ed. Hamilton: Ontario, 2000;262-73.

20. Strauss GM, Gleason RE, Sugarbaker DJ. Screening for lung cancer. Another look; a different view. Chest 1997;111:754-68.

21. Weiss W, Boucot KR, Cooper DA. The Philadelphia Pulmonary Neoplasm Research Project: survival factors in bronchogenic carcinoma. JAMA 1971;216:2119-23.

22. Weiss W, Boucot KR. The Philadelphia Pulmonary Neoplasm Research Project: early roentgenographic appearance of bronchogenic carcinoma. Arch Intern Med 1974;134:306-11.

23. Boucot KR, Weiss W. Is curable lung cancer detected by semiannual screening? JAMA 1973;224:1361-5.

24. Lilienfeld A, Archer PG, Burnett $\mathrm{CH}$. An evaluation of radiologic and cytologic screening for the early detection of lung cancer: a cooperative pilot study of the American Cancer Society and the Veterans Administration. Cancer Res 1966;26:2083-121.

25. Hayata Y, Funatsu H, Kato H, Saito Y, Sawamura K, Furose K. Results of lung cancer screening programs in Japan. Recent results Cancer Res 1982;82:163-73

26. Nash FA, Morgan JM, Tomkins JG. South London lung cancer study. BMJ 1968;2:715-21.

27. Brett GZ. The value of lung cancer detection by six-monthly chest radiographs. Thorax 1968;23:414-20.

28. Brett GZ. Earlier diagnosis and survival in lung cancer. BMJ 1969;4: 260-2.

29. Wilde J. A 10-year follow-up of semi-annual screening for early detection of lung cancer in the Erfurt County, GDR. Eur Respir J 1989;2: 656-62.

30. Ginsberg RJ, Hill LD, Eagan RT, Thomas P, Mountain CF, Deslauriers $\mathrm{J}$, et al. Modern 30-day operative mortality for surgical resections in lung cancer. J Thorac Cardiovasc Surg 1983;86:654-7.

31. Sobue T, Moriyama N, Kaneko M, Kosumoto M, Kobayashi T, Tsuchiya $\mathrm{R}$, et al. Screening for lung cancer with low-dose helical computed tomography: anti-lung cancer association project. J Clin Oncol 2002; 20:911-20

32. Kaneko M, Eguchi K, Ohmatsu H, et al. Peripheral lung cancer: screening and detection with low-dose spiral CT versus radiography. Radiology 1996;201:798-802.

33. Sone S, Takashima S, Li F, Yang Z, Honda T, Maruyama Y, et al. Mass screening for lung cancer with mobile spiral computed tomography scanner. Lancet 1998;351:1242-5.

34. Henschke CI, McCauley DI, Yankelevitz DF, Naidich DP, McGuiness G, Miettinem OS, et al. Early lung cancer action project: overall design and findings from baseline screening. Lancet 1999;354:99-105.

35. Hillman BJ, Gatsonis C, Sullivan DC. American College of Radiology imaging network: new national cooperative group for conducting clinical trials of medical imaging technologies. Radiology 1999;213:6415.

36. Szabo E, Birrer MJ, Mulshine JL. Early detection of lung cancer. Semin Oncol 1993;20:374-82

37. Tockman MS, Mulshine JL, Piantadosi S, Erosan YS, Gupta PK, Ruckdeschel JL, et al. Prospective detection of preclinical lung cancer: results from two studies of heterogeneous nuclear ribonucleoprotein A2/ B1 overexpression. Clin Cancer Res 1997;3:2237-46.

38. Fong KM, Sekido Y, Minna JD. Molecular pathogenesis of lung cancer. J Thorac Cardiovasc Surg 1999;118:1136-52.
39. Lam S, Shibuya H. Early diagnosis of lung cancer. Clin Chest Med 1999;20:53-61.

40. Mulshine JL, Zhou J, Treston AM, Szabo E, Tockman MS, Cuttitta F. New approaches to the integrated management of rarly lung cancer Hematol Oncol Clin North Am 1997;11:235-52.

41. Jacobson DR, Fishman CL, Mills NE. Molecular genetic tumor markers in the early diagnosis and screening of non-small-cell lung cancer. Ann Oncol 1995;6(Suppl 3):S3-S8.

42. Tockman MS, Gupta PK, Myers JD, Frost JK, Baylin SB, Gold EB, et al. Sensitive and specific monoclonal antibody recognition of human lung cancer antigen on preserved sputum cells: a new approach to early lung cancer detection. J Clin Oncol 1988;6:1685-93.

43. Crowell RE, Gilliland FD, Temes RT, Harms HJ, Neft RE, Heaphy E, et al. Detection of trisomy 7 in nonmalignant bronchial epithelium from lung cancer patients and individuals at risk for lung cancer. Cancer Epidemiol Biomarkers Prev 1996;5:631-7.

44. Lam S, Kennedy T, Unger M, Millery E, Gelmont D, Rusch V, et al. Localization of bronchial intraepithelial neoplastic lesions by fluorescence bronchoscopy. Chest 1998;113:696-702.

45. Ikeda N, MacAulay C, Lam S, LeRiche J, Paye P, Garner D, et al. Malignancy associated changes in bronchial epithelial cells and clinical applications as a biomarker. Lung Cancer 1998;19:161-6.

46. Grannis Jr FW. Lung cancer overdiagnosis bias: "The Gyanousa am Loose!". Chest 2001;119:322-3.

47. Kramer BS, Brown ML, Prorok PC, Potosky AL, Gohagan JK. Prostate cancer screening: what we know and what we need to know. Ann Intern Med 1993;119:914-23.

48. McFarlane MJ, Feinstein AR, Wells CK. Necropsy evidence of detection bias in the diagnosis of lung cancer. Arch Intern Med 1986;146: 1695-8.

49. McFarlane MJ, Feinstein AR, Wells CK. Clinical features of lung cancers discovered as a postmortem "surprise". Chest 1986;90:520-3.

50. McFarlane MJ, Feinstein AR, Wells CK, Chan CK. The "epidemiologic necropsy". Unexpected detections, demographic selections, and changing rates of lung cancer. JAMA 1987;258:331-8.

51. Muhm JR, Miller WE, Fontana RS, Sanderson DR, Uhlenhopp MA Lung cancer detected during a screening program using four-month chest radiographs. Radiology 1983;148:609-15.

52. Quekel LG, Kessels AG, Goei R, van Engelshoven JM. Miss rate of lung cancer on the chest radiograph in clinical practice. Chest 1999 ; 115:720-4.

53. Sone S, Li F, Yang ZG, Takashima S, Maruyama Y, Hasegawa M, et al. Characteristics of small lung cancers invisible on conventional chest radiography and detected by population based screening using spiral CT. Br J Radiol 2000;73:137-45.

54. Kaneko M, Kusumoto M, Kobayashi T, Moriyama N, Naruke T, Ohmatsu $\mathrm{H}$, et al. Computed tomography screening for lung carcinoma in Japan. Cancer 2000;89:2485-8.

55. Leef JL, Klin JS. The solitary pulmonary nodule. Radiol Clin North Am 2002;40:123-43.

56. Henschke CI, McCauley DI, Yankelevitz DF, Naidich DP, McGuinness $G$, Miettinen OS, et al. Early lung cancer action project: a summary of the findings on baseline screening. Oncologist 2001;6:147-52.

57. Patz Jr EF, Rossi S, Harpole DH, Herndon JE, Goodman PC. Correlation of tumor size and survival in patients with stage IA non-small cell lung cancer. Chest 2000;117:1568-71.

58. Black WC. Unexpected observations on tumor size and survival in stage IA non-small cell lung cancer. Chest 2000;117:1532-4.

59. Heffner JE, Silvestri G. Editorials. CT screening for lung cancer - Is smaller better? Am J Respir Crit Care Med 2002;165:433-4.

60. Swensen SJ, Jett JR, Sloan JA, Midthum DE, Hartman TE, Sykes AM, et al. Screening for lung cancer with low-dose spiral computed tomography. Am J Respir Crit Care Med 2002;165:508-13.

61. Mountain CF. Revisions in the international system for staging lung cancer. Chest 1997;111:1710-7. 